\title{
MR-Mirror: A Complex of Real and Virtual Mirrors
}

\author{
Hideaki Sato, Itaru Kitahara, and Yuichi Ohta \\ Department of Intelligent Interaction Technologies, \\ Graduate School of Systems and Information Engineering, University of Tsukuba, \\ Tennodai 1-1-1, Tsukuba, Ibaraki, 305-8573, Japan \\ hsato@image.iit.tsukuba.ac.jp, \\ \{kitahara, ohta\} @iit.tsukuba.ac.jp
}

\begin{abstract}
MR-mirror is a novel Mixed-Reality (MR) display system created by using real and virtual mirrors. It merges real visual information reflected on a real mirror and a virtual one displayed on an electronic monitor. A user's body is presented by the reflection on the real mirror, and virtual objects are presented on a monitor that is visible through the real mirror. Users can observe an MR scene without wearing such devices as a head-mounted display and can interact with the virtual objects around them using their body motion in MR space. We implemented a prototype MR-mirror and a demonstration system.
\end{abstract}

Keywords: Mixed Reality, Virtual Mirror, Interaction.

\section{Introduction}

Mixed Reality (MR) is expected to develop novel user interfaces in such fields as medical welfare (rehabilitation), Computer Supported Cooperative Work (CSCW), education, and entertainment [1][2]. To realize such an advanced interface system, we have to consider immersive interaction in MR space that exploits user body motions. Body motion is important to express nonverbal information and to interact with real and virtual 3D objects in MR space.

Due to size and weight reductions, HMDs have become the most popular display system to observe all-around MR scenes [3]. However they need wired cables to supply electronic power and to transmit video signals. As a result, the user motions and the movable areas are limited by the length and weight of the cables. The user's field of view is determined by the HMD specifications, which however are generally not enough wide to cover the whole field-of-view of human eyes.

Projector-based MR systems display MR scenes using video projectors to cast the appearance of virtual objects onto the real world [4]. Since they make it possible to observe MR scenes without wearing any electronic devices, user motions are not restricted. However, displaying 3D virtual objects floating in midair is difficult.

This paper proposes our novel method, "MR-mirror," to realize immersive interaction in MR space. The overview of our MR-mirror is illustrated in figure 1 . In the MR-mirror system, users observe the real world in a half mirror set in front of them. At the same time, they observe the virtual world in a monitor through a half mirror (virtual mirror technique [5]). By reproducing the optical characteristics of real mirrors on the virtual mirror, users can observe MR scenes. In the MR-mirror system, 


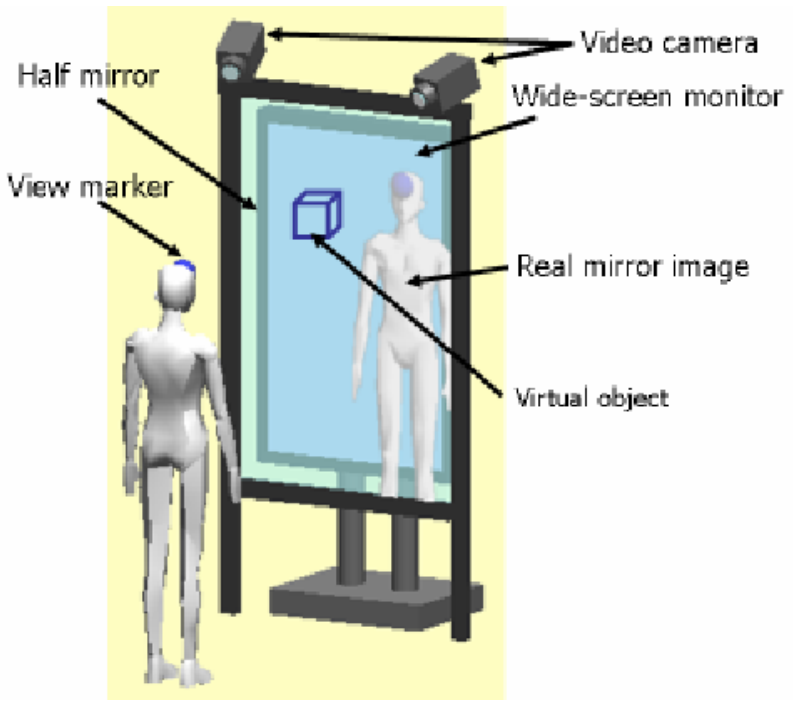

Fig. 1. Overview of MR-mirror system

users do not have to wear any electronic devices, and 3D virtual objects can be observed in the virtual mirror. Our system has another advantage: it can present the user's appearance without any calculation cost, since it is done by a natural phenomenon called specular reflection on the real mirror.

\section{MR-Mirror System}

As shown in figure 1, an MR-mirror system consists of a half mirror to reflect the real mirror image and a wide-screen monitor to show the virtual image. A view marker and two video cameras are used to calculate the user's viewing position necessary for generating the virtual mirror image. The two cameras, which are set at the top of the monitor, capture the user's body in front of the system.

Figure 2 shows the processing flow of the MR-mirror system. As a preparatory offline process, we conducted camera calibration to estimate the intrinsic and extrinsic parameters of the stereo video cameras. Perspective projection matrixes of the two cameras are calculated with the parameters. The system extracts a view marker attached to the user's head in the captured stereo images and estimates the 3D position using the stereo-vision technique. By using the estimated 3D position of the view marker, the system calculates the virtual camera's position to generate mirror images of virtual objects. The virtual mirror images are displayed on the monitor. Finally, an "MR-mirror" is realized with which users can interact with virtual objects by watching their body motion in a real mirror. 


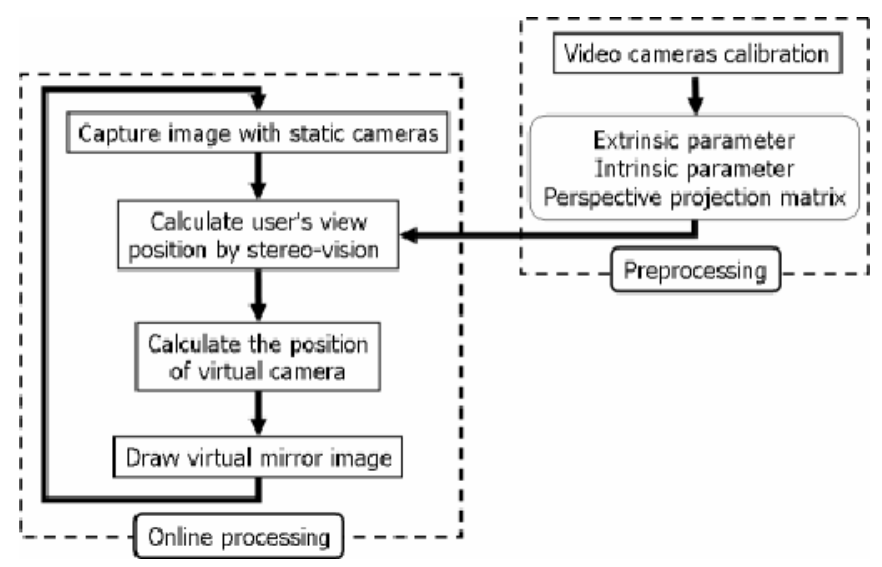

Fig. 2. Processing flow of MR-mirror system
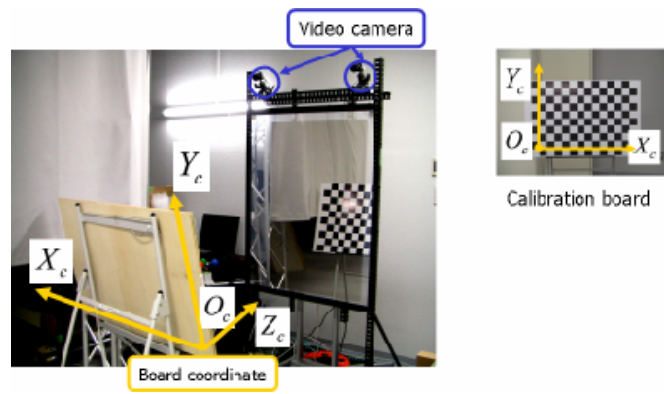

Calibration board

Fig. 3. Calibration of video cameras

\section{Camera Calibration}

Figure 3 shows an overview of the camera calibration procedure and the coordinate systems in the MR-mirror system. Using Zhang's camera calibration method [6], we calculated the intrinsic and extrinsic parameters of the video cameras based on board coordinate system $\boldsymbol{W c}$. The origin $\boldsymbol{O c}$ of the coordinate system is at the bottom left corner of the calibration board. The $\boldsymbol{X c}$-axis is defined as the upward direction of the board's left side edge, and the $Y \boldsymbol{c}$-axis is defined as the right direction of the bottom edge. The $\boldsymbol{Z c}$-axis is defined by calculating the outer product of the $\boldsymbol{X} \boldsymbol{c}$ and $\boldsymbol{Y c}$ axes. As a result, the projective transformation is estimated, as shown in Eq. (1). Here, 3D point $(X c, Y c, Z c)$ in $\boldsymbol{W c}$ is observed at $(u, v)$ in a video camera image, and $\boldsymbol{P c}$ is the projective matrix:

$$
s(u, v, 1)^{T}=P_{c}\left(X_{c}, Y_{c}, Z_{c}, 1\right)^{T} .
$$




\section{3D Position Estimation of a Marker Using Stereo-Vision}

The video cameras were calibrated as described above. The 3D position of a captured marker can be estimated with stereo-vision. The projective relationship between a 3D point $M c\left(x_{c}, y_{c}, z_{c}\right)$ and observing point $m=[u, v]^{T}$ in an image is described in Eq. (2):

$$
s \bar{m}=P_{c} \bar{M}_{c} .
$$

As illustrated in Fig. 4. Stereo-vision

when 3D point $\boldsymbol{M c}$ is observed at $m^{\prime}=\left[u^{\prime}, v^{\prime}\right]^{T}$ in the other image, we have Eq. (3):

$$
s^{\prime} \bar{m}^{\prime}=P_{c}^{\prime} \bar{M}_{c} \text {. }
$$

Here, $\bar{m}, \overline{m^{\prime}}$, and $\bar{M} c$ are the homogeneous vectors of $m, m^{\prime}$, and $M c$, respectively. $s$ and $s^{\prime}$ are scalar with depth values and $P$ and $P^{\prime}$ are the projection matrices of the video cameras .

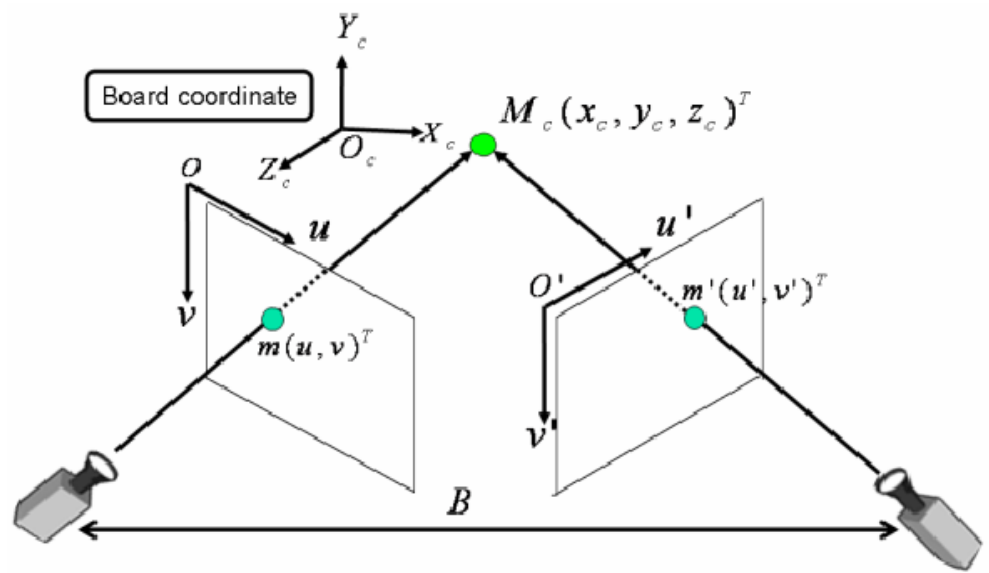

Fig. 4. Stereo-vision

Equation (4) is derived by merging Eqs. (2) and (3). $p_{i j}$ and $p_{i j}^{\prime}$ are elements of projective matrices $P^{\prime} c$ and $P_{c}$.

$$
\begin{gathered}
B M_{c}=b \\
B=\left[\begin{array}{ccc}
u p_{31}-p_{11} & u p_{32}-p_{12} & u p_{33}-p_{13} \\
u p_{31}-p_{21} & u p_{32}-p_{22} & u p_{33}-p_{23} \\
u p_{31}^{\prime}-p_{11}^{\prime} & u p_{31}^{\prime}-p_{11}^{\prime} & u p_{31}^{\prime}-p_{11}^{\prime} \\
u p_{31}^{\prime}-p_{11}^{\prime} & u p_{31}^{\prime}-p_{11}^{\prime} & u p_{31}^{\prime}-p_{11}^{\prime}
\end{array}\right] \quad b=\left[\begin{array}{c}
p_{14}-u p_{34} \\
p_{24}-u p_{34} \\
p_{14}^{\prime}-u p_{34}^{\prime} \\
p_{24}^{\prime}-u p_{34}^{\prime}
\end{array}\right]
\end{gathered}
$$


When a pair of two corresponding points $\boldsymbol{m}$ and $\boldsymbol{m}$ ' in the video camera images is known, we have four linear independent simultaneous equations. However, the number of unknown parameters is three $\left(x_{c}, y_{c}, z_{c}\right)$. In such cases, the simultaneous equations can be solved with pseudo-inverse matrix $\boldsymbol{B}+$, as described in Eq. (5):

$$
M_{c}=B^{+} b \quad B^{+}=\left(B^{T} B\right)^{-1} B^{T} .
$$

\section{Integration of Coordinate Systems}

Figure 5 illustrates the two coordinate systems used in the MR-mirror system. To integrate the captured real world and the 3D CG world, matrix $\boldsymbol{C} \boldsymbol{c w}$, which transforms board coordinate system $\boldsymbol{W} \boldsymbol{c}$ to world coordinate system $\boldsymbol{W}$, must be calculated. In the MR-mirror system, origin $\boldsymbol{P}_{\boldsymbol{w}} \boldsymbol{0}$ of world coordinate system $\boldsymbol{W}$ is defined at the top left corner of the monitor. The $\boldsymbol{X} \boldsymbol{w}$-axis is defined as the right direction of the monitor's upper edge, and the $\boldsymbol{Y} \boldsymbol{w}$-axis is defined as the upward direction of the left side edge. The $\boldsymbol{Z} \boldsymbol{w}$-axis is the outer product of the $\boldsymbol{X} \boldsymbol{w}$ and $\boldsymbol{Y} \boldsymbol{w}$ axes and expresses the depth information of the user's view volume.

First, we measured six 3D points $\{\boldsymbol{P c 0}, \boldsymbol{P} \boldsymbol{c 1}, \boldsymbol{P} \boldsymbol{c} 2\}$ on the calibration board and $\left\{\boldsymbol{P}_{\boldsymbol{w} 0}, \boldsymbol{P}_{\boldsymbol{w} 1}, \boldsymbol{P}_{\boldsymbol{w} 2}\right\}$ on the monitor. Here, $\boldsymbol{P c \boldsymbol { 0 }}$ is at the origin of $\boldsymbol{W c}, \boldsymbol{P} \boldsymbol{c} 1$ is on the $\boldsymbol{X c}$ axis, $\boldsymbol{P} \boldsymbol{c} 2$ is on the $\boldsymbol{Y c}$-axis, $\boldsymbol{P}_{\boldsymbol{w}} \boldsymbol{0}$ is at the origin of $\boldsymbol{W}, \boldsymbol{P}_{\boldsymbol{w}} \boldsymbol{I}$ is on the $\boldsymbol{X} \boldsymbol{w}$-axis, and $\boldsymbol{P}_{\boldsymbol{w}} \boldsymbol{z}$ is on the $\boldsymbol{Y} \boldsymbol{w}$-axis. Translation vector $\boldsymbol{T} \boldsymbol{c} \boldsymbol{w}$ is defined by calculating $\boldsymbol{P} \boldsymbol{c o} \boldsymbol{0} \boldsymbol{P} \boldsymbol{P} \boldsymbol{w}$. The basis

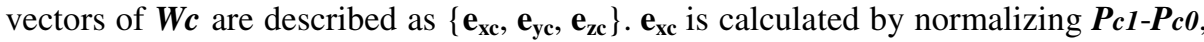
and $\mathbf{e}_{\mathrm{yc}}$ is calculated by normalizing $\boldsymbol{P c 2}-\boldsymbol{P c} \boldsymbol{0} . \mathbf{e}_{\mathrm{zc}}$ is a normalized cross product of $\mathbf{e}_{\mathrm{xc}}$ and $\mathbf{e}_{\mathbf{y c}}$. The basis vectors of $\boldsymbol{W}$ are described as $\left\{\mathbf{e}_{\mathbf{x w}}, \mathbf{e}_{\mathbf{y w}}, \mathbf{e}_{\mathbf{z w}}\right\} . \mathbf{e}_{\mathbf{x w}}$ is calculated by normalizing $\boldsymbol{P}_{\boldsymbol{w}} \boldsymbol{1}-\boldsymbol{P} \boldsymbol{w} \boldsymbol{0}$, and $\mathbf{e}_{\mathrm{yw}}$ is calculated by normalizing $\boldsymbol{P}_{\boldsymbol{w} \mathbf{2}}-\boldsymbol{P} \boldsymbol{w} \boldsymbol{0} . \mathbf{e}_{\mathbf{z w}}$ is a normalized cross product of $\mathbf{e}_{\mathbf{x w}}$ and $\mathbf{e}_{\mathbf{y w}}$. 3D rotation matrix $\boldsymbol{R} \boldsymbol{c} w$ is defined by the basic vectors. As shown in Eq. (6), $\boldsymbol{C} \boldsymbol{c} w$ is given by composing $\boldsymbol{T} \boldsymbol{c} w$ and $\boldsymbol{R} \boldsymbol{c} w$ :

$$
\left[\begin{array}{l}
e_{x w} \\
e_{y w} \\
e_{z w}
\end{array}\right]^{T}=R_{c w}\left[\begin{array}{l}
e_{x c} \\
e_{y c} \\
e_{z c}
\end{array}\right]^{T} \quad T_{c w}=P_{c 0}-P_{w 0} \quad C_{c w}=\left[\begin{array}{cc}
R_{c w} & T_{c w} \\
0 & 1
\end{array}\right] .
$$

With calculated transformation matrix $\boldsymbol{C} \boldsymbol{c} w$, the estimated 3D position of marker $\boldsymbol{M c}$ is projected to $\boldsymbol{M}$ in the world coordinate system:

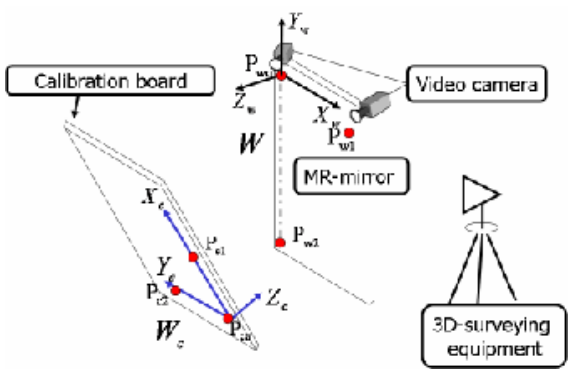

Fig. 5. Relation between two coordinate systems 


\section{Virtual Mirror Image Generation}

\subsection{Layout of a Virtual Camera and Virtual Objects}

Figure 6 shows the geometrical relationship between the real and virtual worlds of the "MR-mirror". Although we only use the $X_{w}$-axis and the $Z_{w}$-axis to simply explain the geometry, the same relationship can be extended to the 3D world. When there is a real object at $\boldsymbol{V}_{\boldsymbol{o b j}}$, and a user's viewpoint at $\boldsymbol{V}_{\boldsymbol{v}}$, objects are observed along with the solid line (line of sight) reflected on a mirror, as shown in Fig. 6. Geometric relation between real and virtual worlds in MR-mirror.

This optical phenomenon can be expressed without mirror reflection. In this case, the object is assumed to exist at $\boldsymbol{V}_{o b j}^{\prime}$, where symmetry exists with the plane of the mirror, and the appearance is seen through the mirror along with the dotted line. The optical geometry is basically the same in both the real and virtual worlds. So generating a virtual mirror image is possible that displays a virtual object at $\boldsymbol{V}_{\boldsymbol{o b j}}$ by setting the virtual camera $\left(\boldsymbol{V}_{c a m}\right)$ at $\boldsymbol{V}_{v}$ and the object at $\boldsymbol{V}_{o b j}^{\prime}$. In an MR-mirror 3D coordinate system, the mirror plane coincides with the $\boldsymbol{X} \boldsymbol{w}-\boldsymbol{Y w}$ plane. Thus, it is easy to generate virtual objects that have symmetry with the mirror to inverse the sign of the $\mathbf{Z w}$ (depth) value of the $3 \mathrm{D}$ data.

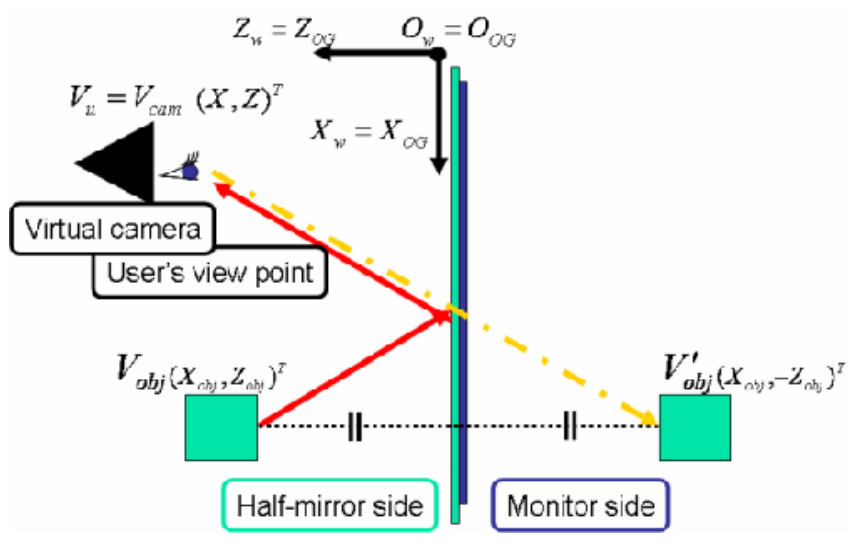

Fig. 6. Geometric relation between real and virtual worlds in MR-mirror

\subsection{View Volume of a Virtual Camera}

Since the observable region of the MR-mirror is limited by the monitor's area, rendering all virtual objects is not efficient, even out of the displaying area. Fig. 7. View volume of a virtual camera shows the change of the observable area (view volume) when a virtual camera moves from $\boldsymbol{V}_{\text {cam }}$ to $\boldsymbol{V}_{\text {cam }}^{\prime}$. To render only observable virtual objects, the MR-mirror system sets the view volume of a virtual camera depending on the monitor area and viewpoint $\boldsymbol{V}_{\text {cam }}$. As illustrated in Fig. 7. View volume of a 


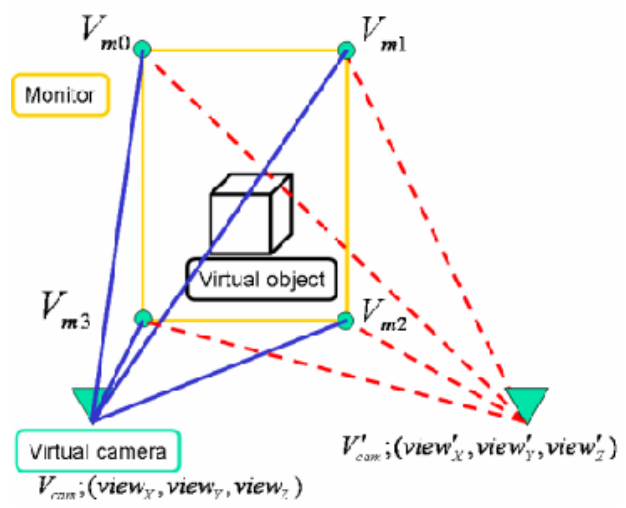

Fig. 7. View volume of a virtual camera

virtual camera, the 3D coordinates of the four vertices of the monitor are $\boldsymbol{V m \boldsymbol { m }}, \boldsymbol{V m} \mathbf{1}$, $V m 2$, and $V m 3$. Then the view volume is set as a square pyramid with a base comprised of $V_{m} 0, V m 1, V m 2$, and $V m \mathbf{3}$ and a cone point set as $V_{\text {cam }}$. When the virtual camera moves, the square pyramid is deformed by moving the cone point. On the other hand, the base is fixed on the monitor.

\section{Implementation of an MR-Mirror System}

\subsection{Specifications}

Figure 8 shows the pilot system of the MR-mirror. All processes are executed by a single computer with two Intel Core2Duo processors $(2.13 \mathrm{GHz})$, a 4-GB memory system, and a video card (nVidia "GeForce7900GS"). Generated virtual mirror images are displayed on a large Liquid Crystal Display (LCD) monitor (SHARP "PN455R": 45 inches) that can display 1920 [pixel] $\times 1080$ [pixel] images and whose display size is $985.9[\mathrm{~mm}] \times 554.6[\mathrm{~mm}]$. We use this monitor with portrait style. IEEE1394b video cameras (Point Grey Research "Flea2"), which can capture 1024 [pixel] $\times 768$ [pixel] images with 30 [fps], are used for detecting markers.

\subsection{Evaluation}

We experimentally evaluated the feasibility of our proposed method. First, we confirmed whether the real and virtual objects are correctly overlapped on the MR-mirror. In the evaluation a real object, a gypsum cube, was set in front of the MR-mirror, as shown in Figure 8. A virtual object, a wire-frame cube with the same shape as the real object, was also set on the same position in $3 \mathrm{D}$ space. Instead of observation by a user, we put a digital camera to capture the sight. Figure 9 shows the images captured by the digital camera by moving it from right to left or up and down. We confirmed that the appearance of the virtual object is overlapped on the real object and that the geometry of the MR-mirror is adequately realized. 


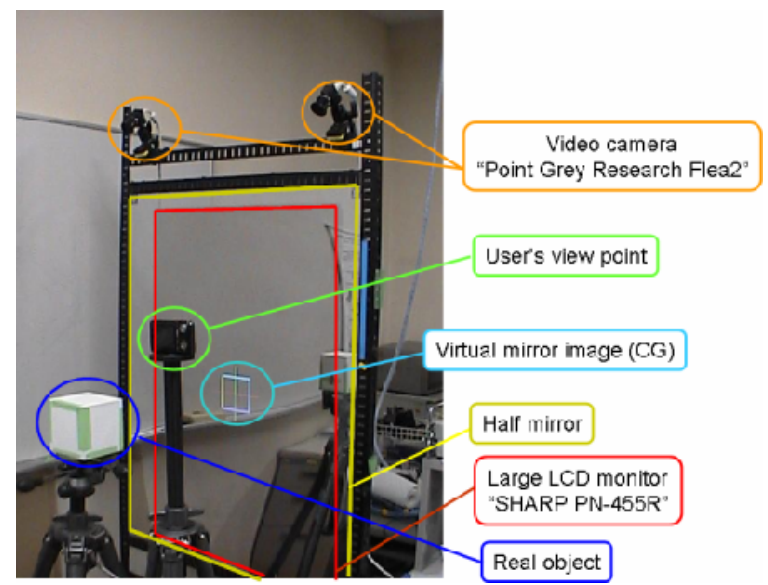

Fig. 8. Prototype system of MR-mirror
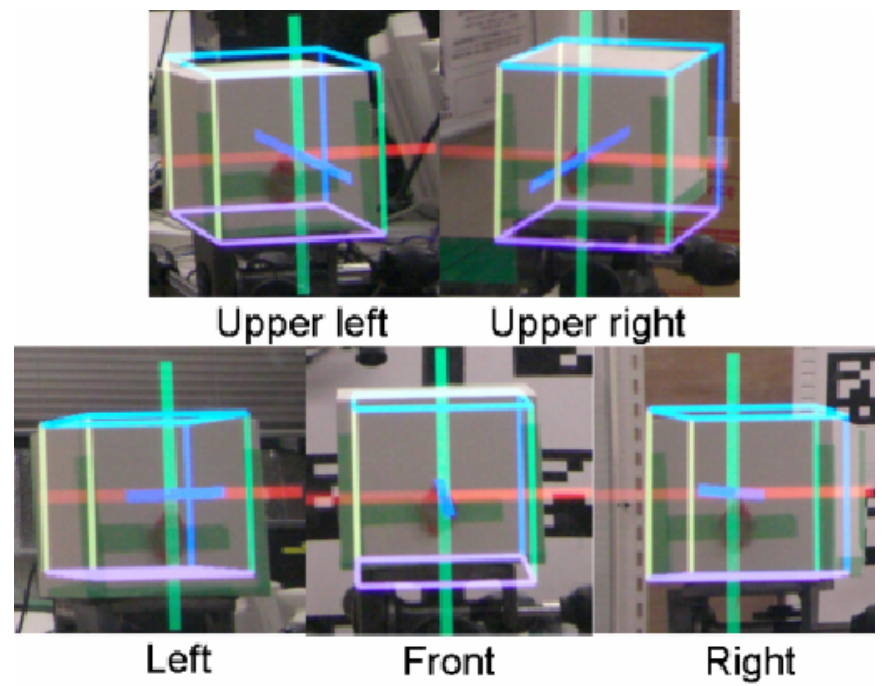

Fig. 9. Experimental results. Appearance of virtual object is overlapped on real object.

\section{Game for MR-Mirror Demonstration}

We developed a demonstration game system utilizing the effectiveness of the MRmirror. Figure 10 shows the demonstration system. A player stands in front of the MR-mirror with two colored markers. The one on the player's head is a view marker to detect the viewpoint, and the other is an interaction marker to make contact with the virtual objects. In the MR scene (figure 10), some virtual objects (moving balls) are observed. The purpose of the game is to flick out the balls using body motion. The 
player moves the interaction marker to where a virtual object exists while observing the MR-mirror. When the interaction marker hits the ball in the MR scene, it is flicked out.

Since we are used to looking at mirrors in our daily life, we can easily understand the geometrical relationship between the real and virtual objects. To flick out all the balls, the player has to make large body motion. This suggests that the MR-mirror might be used for exercising applications, such as rehabilitation or the maintenance of good health.

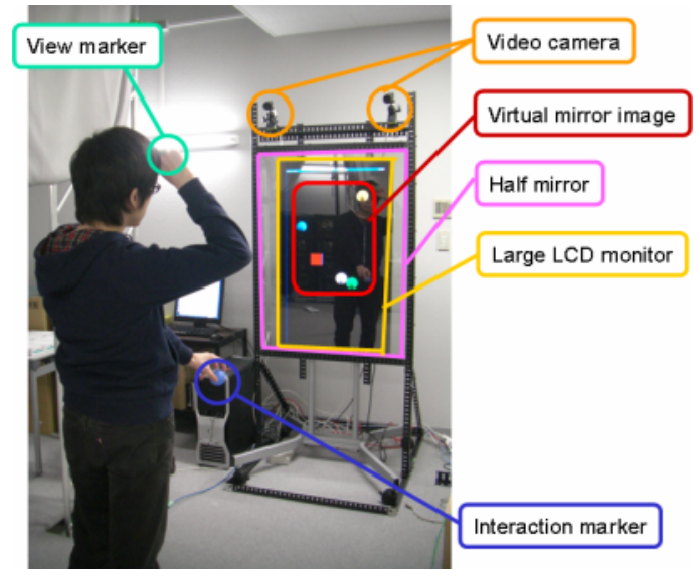

Fig. 10. Game for MR-mirror demonstration

\section{Conclusion}

This paper proposed an MR display method called "MR-mirror" that can display 3D virtual objects in midair without any wearable display devices. In the MR-mirror system, the appearance of real and virtual worlds is mixed using a half mirror. By integrating the $3 \mathrm{D}$ coordinate system between the real and virtual worlds and setting virtual objects to generate virtual mirror images, a user can observe an immersive MR scene without an HMD. We developed a prototype MR-mirror system to confirm its feasibility and effectiveness. Future works include the elimination of the half mirror's distortion or motion capture without colored markers. However, we confirmed the features of the MR-mirror with the developed system.

\section{References}

1. Minatani, S., Kitahara, I., Kameda, Y., Ohta, Y.: Face-to-Face Tabletop Remote Collaboration in Mixed Reality. In: ISMAR 2007, pp. 43-46 (2007)

2. Richard, E., Billaudeau, V., Richard, P.: Augmented reality for rehabilitation of disabled children: a preliminary study. In: Virtual Rehabilitation 2007, pp. 102-108 (2007)

3. Ohta, Y., Tamura, H.: Mixed Reality-Merging Real and Virtual Worlds. Ohmsha, Ltd. (1999) 
4. Bimber, O., Raskar, R.: Spatial Augmented Reality Merging Real and Virtual Worlds. A.K. Peters, Ltd. (2005)

5. Ushida, K., Tanaka, Y., Naemura, T., Harashima, H.: i-mirror: An Interaction/ Information Environment Based on a Mirror Metaphor Aiming to Install into Our Life Space. In: Proceedings of the 12th International Conference on Artificial Reality and Telexistence (ICAT 2002) (December 2002)

6. Zhang, Z.: A Flexible new technique for camera calibration. IEEE Transaction on Pattern Analysis and Machine Intelligence (2000)

7. Jo, G., Tsuji, S.: Three dimensional vision (in Japanese), Kyoritsu Shuppan (1998); ISBN13: $978-4320085220$ 